\title{
Too many reviews too few trials
}

\section{Derek Richards}

Editor

This year the Journal reaches its 20th birthday, the first issue having been published in November 1998. Since that time we have been publishing summaries of guidelines, systematic reviews and highquality studies that we have identified through regular searches of potential literature. We have also encouraged people to undertake systematic reviews and provided information on how to appraise them. Over the years systematic reviews have provided the bulk of the articles we have summarised. However, I wonder if now is the time to ask whether we are conducting too many reviews and undertaking too few high-quality original studies.

Let's take as an example a review in this issue which considers the relationship between periodontal disease and periodontal outcomes. ${ }^{1}$ This Cochrane review includes 15 randomised controlled trials, however all were at high risk of bias. As a result the authors concluded it was unclear if periodontal treatment during pregnancy has an impact on preterm birth, although it may reduce low birth weight, with the overall quality of the evidence being judged to be low quality.

Many reviews on this topic have been conducted previously and a basic search of Medline shows 30 review publications on this topic between 2003 and 2017. Many of these highlight a lack of evidence, often pointing out limitations in the available evidence. A second example is that of the surgical management of the keratocyst; again a brief search of Medline finds 16 systematic reviews of this topic between 2000 and 2018 with eight of these reviews published within the past two years. All of these reviews are largely based on retrospective studies with no head to head comparisons of different treatment approaches.

These are but two examples of the growing number of repeated reviews of the same or very similar topics in dentistry often undertaken during a similar time period. This is a potential waste of resources, particularly when, as in the two examples noted above, there are limited good quality primary research studies available to address the questions being asked. For while systematic reviews are valuable in summarising available data and helping identify gaps, there seems to be little added benefit in repeatedly pointing out the same gaps. Successive dental systematic reviews have highlighted either a lack of evidence or only the availability of poor quality evidence to address a number of clinical and research questions. So instead of focusing on repeating systematic reviews we need to place greater emphasis on developing and delivering high quality totally relevant research.

While systematic reviews have identified many gaps in the dental evidence base I hope that many of you will have taken the time to respond to the recent Oral \& Dental Health Priority Setting Partnership Survey (https://oralanddentalhealthpsp. wordpress.com/) here in the UK. This work is being coordinated by the James Lind Alliance (JLA) and the National Institute for Health Research Evaluation, Trials and Studies Coordinating Centre (NETSCC). It is hoped that this initiative will lead to more ambitious collaborative and multicentre working help address some of our key dental questions.

To return to systematic reviews there is still an ongoing need for them to be undertaken where they have not been done previously or where new evidence has been published that requires updates to be undertaken. Some guidance on when systematic reviews should be updated is provided in a 2016 consensus statement. 2 It is also recommended that you check the PROSPERO database (https://www. crd.york.ac.uk/prospero/) which holds protocol details of registered and ongoing systematic reviews to reduce duplication. For example there are currently 542 records relating to periodontal disease and 435 related to orthodontic treatments. If you are considering undertaking a systematic review you should check this database first to help reduce duplication. You can also register your own review.

1. Iheozor-Ejiofor Z, Middleton P, Esposito M, Glenny AM. Treating periodontal disease for preventing adverse birth outcomes in pregnant women. Cochrane Database Syst Rev 2017; 6: Art. No.: CD005297. DOI: 10.1002/14651858.CD005297.pub3.

2. Garner P, Hopewell S, Chandler J, et al., Panel for updating guidance for systematic reviews (PUGs). When and how to update systematic reviews: consensus and checklist. Br Med J 2016; 354: i3507. doi:10.1136/bmj.i3507. Erratum in: $\mathrm{Br}$ Med J 2016; 354: i4853. PubMed PMID: 27443385; PubMed Central PMCID: PMC4955793.

Evidence-Based Dentistry (2018) 19, 2. doi:10.1038/sj.ebd.6401280 